\title{
Crystal structure of dirubidium hydrogenphosphide, $\mathbf{R} \mathbf{b}_{2}[\mathbf{P H}]$
}

\author{
M. Somer, W. Carrillo-Cabrera, E.-M. Peters, K. Peters and H. G. von Schnering
}

Max-Planck-Institut für Festkörperforschung. Heisenbergstraße 1. D-70569 Stuttgart. Germany

Received October 25, 1996, transferred to 1st update of database ICSD in 1998. CSD-No. 402681

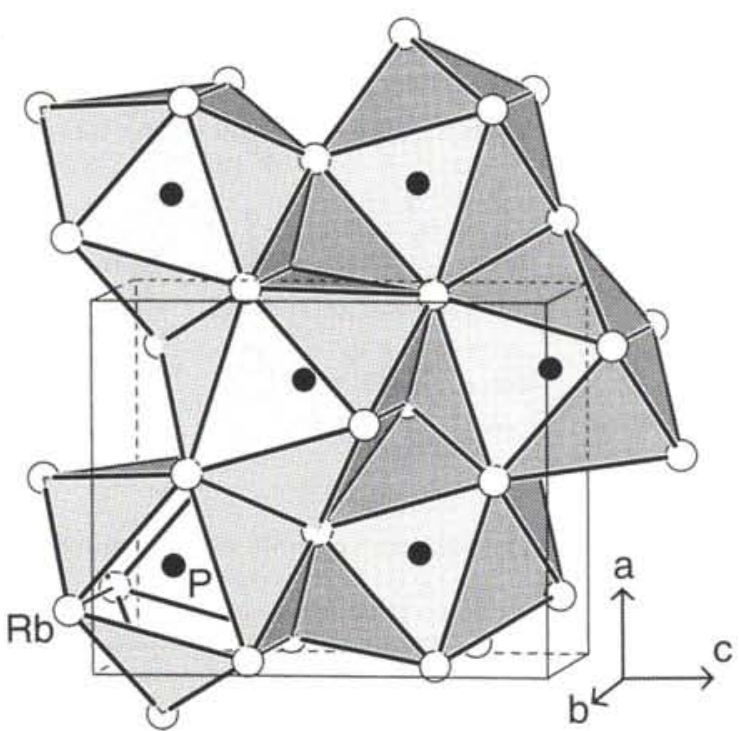

Source of material: Single crystals of $\mathrm{Rb} 2[\mathrm{PH}]$ were first obtained from the reaction of a mixture of $\mathrm{Rb}$ with $\mathrm{HPN}_{2}$ (ratio 5:1) in a sealed steel ampoule at $950 \mathrm{~K}$. The compound can also be synthesized via direct reaction of hydrogen with a mixture of $\mathbf{R b}_{4} \mathrm{P}_{6}$ and $\mathrm{Rb}$ (ratio 1:10) at $950 \mathrm{~K}$. $\mathrm{Rb}_{4} \mathrm{P}_{6}$ and $\mathrm{Rb}$ were placed in a $\mathrm{Nb}$ crucible, which in turn was sealed within a steel ampoule with $\mathrm{ZrH}_{2}$ present as $\mathrm{H}_{2}$ source, but separated from the $\mathbf{R b}_{4} \mathbf{P} 6 / \mathbf{R b}$ mixture. In both cases, excess $\mathrm{Rb}$ was removed at $473 \mathrm{~K}$ in high vacuum.

Table 2. Final atomic coordinates and displacement parameters (in $\AA^{2}$ )

\begin{tabular}{lllllllllll}
\hline Atom & Site & $x$ & $y$ & $z$ & $U_{11}$ & $U_{22}$ & $U_{33}$ & $U_{12}$ & $U_{13}$ & $U_{23}$ \\
\hline $\mathrm{Rb}(1)$ & $4 c$ & $0.8440(2)$ & $1 / 4$ & $0.0769(1)$ & $0.0716(8)$ & $0.0368(6)$ & $0.0364(6)$ & 0 & $-0.0023(6)$ & 0 \\
$\mathrm{Rb}(2)$ & $4 c$ & $0.9787(2)$ & $1 / 4$ & $0.6835(1)$ & $0.0500(7)$ & $0.0605(9)$ & $0.0570(8)$ & 0 & $-0.0051(6)$ & 0 \\
$\mathrm{P}(1)$ & $4 c$ & $0.2470(4)$ & $1 / 4$ & $0.1043(3)$ & $0.051(2)$ & $0.044(2)$ & $0.040(2)$ & 0 & $-0.007(1)$ & 0 \\
\hline
\end{tabular}

\section{References}

1. Rundqvist, S.: The Structures of $\operatorname{Co}_{2} \mathrm{P}, \mathrm{Ru}_{2} \mathrm{P}$ and Related Phases. Acta Chem. Scand. 14 (1960) 1961-1979.

2. Sheldrick, G. M.: Program Package SHELXTL-plus. Release 4.1. Siemens Analytical X-Ray Instruments Inc., Madison (WI 53719), USA 1990.
$\mathrm{Rb}_{2}[\mathrm{PH}]$ crystallizes in the $\mathrm{Co}_{2} \mathrm{P}$ type (see ref. 1). The $[\mathrm{PH}]^{2-}$ anions center the tricapped trigonal prisms built up by the $\mathbf{R b}$ atoms. It was not possible to determine the position of the $\mathrm{H}$ atoms. However, the IR spectra of a single crystal as well as of a powdered sample exbit the characteristic $\mathrm{P}-\mathrm{H}$ absorption at $\mathrm{v}(\mathrm{P}-\mathrm{H})=2122$ $\mathrm{cm}^{-1}$.

HPRb2, orthorhombic, Pnma (No. 62), $a=8.415(1) \AA$,

$b=5.546(2) \AA, c=10.349(2) \AA, V=483.0 \AA^{3}, Z=4, R(F)=0.047$, $R_{\mathrm{w}}(F)=0.046$.

Table 1. Parameters used for the $\mathrm{X}$-ray data collection

$\begin{array}{ll}\text { Crystal: } & \text { orange prism, size } 0.12 \times 0.10 \times 0.13 \mathrm{~mm} \\ \text { Wavelength: } & \text { Mo } K_{\alpha} \text { radiation }(0.71073 \AA) \\ \mu: & 198.50 \mathrm{~cm}^{-1} \\ \text { Diffractometer: } & \text { Siemens P4 } \\ \text { Scan mode: } & \omega \\ \mathrm{T}_{\text {measurement: }} & 293 \mathrm{~K} \\ 2 \theta_{\text {max: }} & 55^{\circ} \\ \mathrm{N}(\text { hkl})_{\text {unique: }} & 611 \\ \text { Criterion for } F_{\mathrm{o}}: & F_{\mathrm{o}}>3 \sigma\left(F_{\mathrm{o}}\right) \\ \text { N(param) } & 20 \\ \text { Programed: } & \text { SHELXTL-plus }\end{array}$

